\title{
The study biochemical indicates of blood rats at conditions acute hypoxia
}

\author{
Serik Abdreshov ${ }^{1,2,{ }^{*}}$, Sultan Tuleukhanov ${ }^{1}$, Gulshat Atanbaeva ${ }^{1}$, Nazym Issayeva $^{1}$, Ainur \\ Zhumabayeva $^{1}$ \\ ${ }^{1}$ Al-Farabi Kazakh National University, al-Farabi Ave. 71, 050040 Almaty, Kazakhstan \\ ${ }^{2}$ Institute of Human and Animal Physiology SK MES RK, al-Farabi Ave. 93, 050060 Almaty, \\ Kazakhstan
}

\begin{abstract}
In the experimental simulation hypoxia, the liver and rhinitis coincide with the discontinuation of rheological organs of the bones and organ lymph, which has diminished in shorter duration of the constriction, increasing the viscosity and thrombogenic processes in the blood and lymph. Unilateral hypoxic hypoxia (1.5 hrs) on liver tissue, decreased blood pressure, decreased intoxication levels in beds at $8 \% . \%$. The concentration of the total protein concentration and the elevation of the activation ALT and AST were investigated by the changes in the osmotic resistance of the erythrocytes and of urine. It has been established that experimental live animals with hypoxia, lymphatic aching, loss of general protein, high levels of alimentary tract and ALT, AST, abnormalities of rheological properties, and lymph nodes and morphophysiological condition of the lymphatic system.
\end{abstract}

\section{Introduction}

One of the stressors for the body is hypoxia and joint hypoxia and hypercapnia. Lack of oxygen in the body as a whole lead to hypoxia, as a result of which the oxidative processes in the tissues are disrupted and the excretion of metabolic products from them is complicated [1]. All-important physiological and biochemical processes in life are largely due to the function of the biological membrane. As a result of the damage to the biomembrane activity, the pathological processes in the body arise. Currently, the literature does not find much information on the structural-functional state of the lymphatic system and nodes in cerebral ischemia, the biochemical composition and rheological properties of member lymph and its connectivity with liquor, the ischemia of the hind limbs of varying duration, and the volume of circulatory fluid in experimental lymphadenopathy [2].

The purpose of the research: to study the biochemical parameters of blood during experimental hypoxia of animals. According to the purpose of the research the following tasks of the work are defined.

Objectives of the research:

- Determination of biochemical indicators of blood in hypoxia in animals.

\footnotetext{
* Corresponding author: ertis_economika@mail.ru
} 
- To study rheological parameters of blood at hypoxia in animals.

Relevance of the research: According to the forecasts of the World Health Organization experts (WHO materials, Geneva, 2003), diseases of the cardiovascular system are widespread in the world in the $21^{\text {st }}$ century. Even in the twentieth century, the incidence of disability and disability has become one of the world's leading socially significant indicators, and these rates will continue in the $21^{\text {st }}$ century.

All-important physiological and biochemical processes in life are largely due to the function of the biomembrane. The cell membranes are sensitive to any damaging effects, and their structural and metabolic modifications can be used to evaluate the functional state of the body. The damaging effects of stress are manifested at the cellular and molecular levels. It is manifested by a violation of the function and structure of the cell membrane, enzyme rejection, irreversible changes in nucleic acids and proteins. Therefore, stress effects on the body and its cell membranes [3].

It has been found that recurrence of stressors, as well as hypoxia, can cause an adaptive response in the body and not only increase its resistance to severe stress effects, such as acute hypoxia, but also protect the body from other damaging factors [4]. In order to increase the functional capacity of the body and increase its resistance to oxygen deficiency and other stress factors, a joint hypoxia and hypercapnic loading method are used [5].

In cardiovascular diseases, severe complications such as heart attack, stroke, congestive endocarditis, and thrombosis can lead to long-term disability and disability. It is known that many cardiovascular diseases lead to the involvement of the lymphatic system in pathological processes, and in turn the lymphatic system can change the course of the disease and the state of its body through its protective and compensatory and transport services [6]. The lymphatic system is involved in compensatory responses to circulatory failure and in circulatory failure and in venous stagnation, vasomotor hypertension, simulation of the effects of weightlessness on the ground, toxic hepatitis, alloxanic diabetes [7].

It is known that oxygen deficiency in the body causes vascular disorders, which results in a decrease in the release of endothelial cells relaxation factors and nitric oxide synthesis, an increase in vascular reactions and vascular permeability, and also a violation of microcirculation [8]. With ischemia, single acidosis develops [9]. In prolonged ischemia, especially in the pre-ischemic period, there is an increase in the thrombogenic properties of blood, an increase in acidosis, free radicals and the development of cellular hyperhydration $[10,11]$. Biochemical indicators of blood and the function of the lymphatic system status in ischemia and oxygen deficiency in organs and tissues are rarely reported in the literature.

In dogs [12] and the lower vertebrates [13], short-term occlusion of the general carotid artery was noted, an increase in lymph flow in the thoracic or intestinal tract. In rats, dogs, and low-lying animals, there was a decrease in lymphatic drainage and lymphatic formation during temporal occlusion of the cranial artery.

However, there is little data on the transport function of the lymphatic vessels and nodes in the neck and hind limbs with ischemia of varying lengths and there is much conflicting evidence. This information was obtained using radiological techniques and contrasting colors. According to some researchers, during acute ischemia, lymphatic vessels in the hind limbs and lymph nodes in the lymph nodes showed an increase in contrast, and in some studies, in dogs and cats, there was a decrease in lymphatic transmission [14].

At present, according to our data and the above-mentioned ischemia of individual limbs, the effects of lymphatic transport on major vessels: the chest or the intestinal tract - have been investigated. However, the literature does not provide much information on the structuralfunctional state of the lymphatic system [10] and nodes in cerebral ischemia [12], the biochemical composition and rheological properties of member lymph and its connectivity with liquor, the ischemia of the hind limbs of varying duration, and the amount of blood flow during experimental lymphadenopathy $[15,16]$. 
Due to this, the lymphatic system has theoretical and practical significance for the defense-compensatory function [11] and its drainage function in various tissues of the body, so that in ischemia of organs and tissues, individual or regional members do not have data on the process of lymph accumulation and the function of lymphatic transport in the body [17]. In this regard, the study of the rheological properties of the lymphatic system, blood and lymph is of interest to many scientists today. Currently, the number of environmental factors which are harmful to the organism is increasing every year [18, 19].

Scientific novelty of the work. In experimental animal's hypoxia revealed a decrease in lymphatic drainage, total protein, an increase in the activity of the ALAT and AcAT enzymes, and a violation of the pulmonological function of lymph and blood; and decreased transport function of the lymphatic system.

Theoretical and practical value of the work: The results of the degree work have high theoretical and practical value and are of great importance for the fundamental physiology and lymphology sciences. According to the data obtained during experimental hypoxia, the involvement of the lymphatic system in the transport, compensatory and adaptive reactions and the role in the physiology of the lymphatic system is high. It has practical significance.

Object of study: Rats, dogs, lymph, blood, blood vessels, lymph nodes and nodules.

\section{Materials and Methods}

The research was carried out in the laboratory of physiology of lymphatic systems of the Institute of Human and Animal Physiology of the Ministry of Education and Science of the Republic of Kazakhstan.

Research methods: The experiment was performed on 6 adult dogs weighing 10-12 kg (narcosis: $35-45 \mathrm{mg} / \mathrm{kg}$ ether or thiopental sodium) experimental hypoxia by binding the arteries to sleep for a period of 30-60 minutes, sometimes lasting 3 hours. Weight hypoxia hypoxia 220-250 g. were performed on 40 laboratory white rats. Experimental hypoxia was maintained in a gas environment for 1.5 hours at $8 \%$ oxygen and $8 \% \mathrm{CO} 2$ in a closed space. Carbon dioxide was absorbed to obtain pure hypoxia.

Studied lymph flow through the lymphatic microcannula channel around the neck of the roots dogs. Biochemical indicators of lymph, the glucose level was determined by method of Reitman-Frenkel with the test strip "Glucotrend-2", amiloliticescoy method, the content of $\alpha$-amylase, alanine aminotransferase (Alat) and aspartate aminotransferase (AST) by the method of Reitman-Frenkel method bilirubin of Jendrasik of GAFTA samples of thymolthymol-veronal buffer, total protein berety method determination of urea systematic method diacetylbenzene identified using clinical diagnostic "Bio-lachema-test" (Czech Republic) when the color reaction, creatinine-Jaffa pirineu acid .

Blood cell morphology was identified in erythrocytes, leukocytes and platelets in the Sismex KX-21 - hematological analyzer (Japan) and in the blood oxygen level and pH indicator and ions in the OSMETECH OPTITM CCA - analyzers (USA). Physico-chemical parameters of blood and lymph were determined: the time of blood and lymph clotting was determined by the Sukharev method, their viscosity in the viscose VK-4, and the hematocrit method.

The physico-chemical parameters of blood and lymph were determined, Sukapev drowsiness was maintained, and VK-4 viscosity was blocked, and the hematopoietic mass method was determined. Blood plasma, lymph and urine were detectedelectrolytically with the Radiometer FPMA ABL615 / 625 assay.

We investigated the spontaneous and induced contractile activity of the isolated blood and lymphatic vessels using a universal method (12) in a 6MX1C mechanotron, chamber, and a recording device - the $\mathrm{H} 339$ and $\mathrm{H} 3012$ milliammeter. As a nutrient solution for roots, Krebs' solution of oxygen was used: $\mathrm{NaCl}$-133.0; NaHCO3-16.3; NaH2PO4-1.38; KCl-5; 
$\mathrm{CaCl} 2$-2.5; $\mathrm{MgCl} 2-0.1$; glucose $-7.8 \mathrm{mM} /$ liter, $\mathrm{pH}-7.4 \mathrm{t}+37^{\circ} \mathrm{C}$. We oxidized the nutrient solution with a gas mixture of $95 \% \mathrm{O} 2$ and $5 \% \mathrm{CO} 2$. The concentration of vasoactive adrenaline, acetylcholine, and histamine $(1 \times 10-8-1 \times 10-3 \mathrm{M})$ was used as a test to measure the excited contractile activity of blood and lymph vessels.

For the study of bloodblood vessels conducted immoderately carotid artery of animals, which was carried out by means of reogepatografiya and regenerate using tetrapolar selection using a rheograph of REO, allowing to completely eliminate the polarization effect of the electrodes on the measurement results. For the examination, we used data from differential rheograms that characterize the rate of change in the blood supply of the studied area.Brain tissue hematopoiesis was determined by Mizar-REO method by rheography method, and volume velocity was determined by Sonomed-300 according to Doppler method. This method is used to determine blood flow velocity in the case of short-term and recurrent ischemia.

The resulting results were calculated using the Microsoft Excel program, including the results of the ratios of the apithmetric mean value, the quadratic octal deviation, the threedimensional apithmetic ratio. Fissep-Student's kite was recalled, and the papuleplet was assumed to be sound when the value was $\mathrm{p} £ 0.05$.

\section{Results and Discussion}

Complete oxygen supply to tissues is one of the key factors behind the evolution of the cardiovascular system. The development of the animal world is largely due to the presence of a high level of cardiovascular system that supplies the tissues of the functioning organs and transports the metabolic products. Providing tissues with adequate oxygen during the evolution of vertebrate animals underlie the structural and functional formation of the cardiorespiratory system [20].

In rats, blood cells during hypoxia, their rheological properties, and hematological parameters were investigated. The animals were divided into three groups, the 1 st control group (10 rats), the $2^{\text {nd }}$ experimental group (15 rats), and the 3 rd experimental group (15 rats) studies for hypoxia. The results of studies performed on rats indicate that mixed acidosis was present during the hypoxia modeling phase. The levels of total carbon dioxide (tCO2) were normally $17.3 \mathrm{mmol} / \mathrm{L}$ (Fig. 1), and during acute hypoxia in rats these values increased by 57.8 and $88.44 \%$.

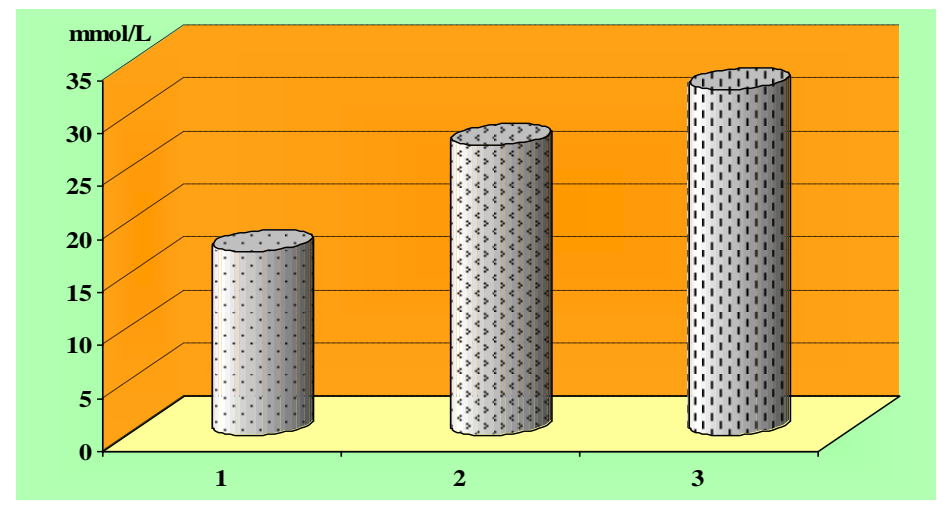

Note: on ordinate axis: blood carbon, mmol / L, on abscess growth: 1 - normal, 2 - hypoxia in 30 minutes, 2 - in hypoxia after 60 minutes.

Fig. 1. Levels of total carbon dioxide (tCO2) concentrations in rats. 
During experimental studies, a slowdown in blood flow results in a lack of oxygen in the brain and blood vessels. In general hypoxia, primary acidosis occurs, resulting in the production of free radicals. The $\mathrm{pH}$ values are important in the organisms of humans and animals. The $\mathrm{pH}$ of the blood showed a $1.8 \%$ decrease $(\mathrm{p}<0.05)$ compared with the control group (pH 7.45 in the control group) (Fig. 2).

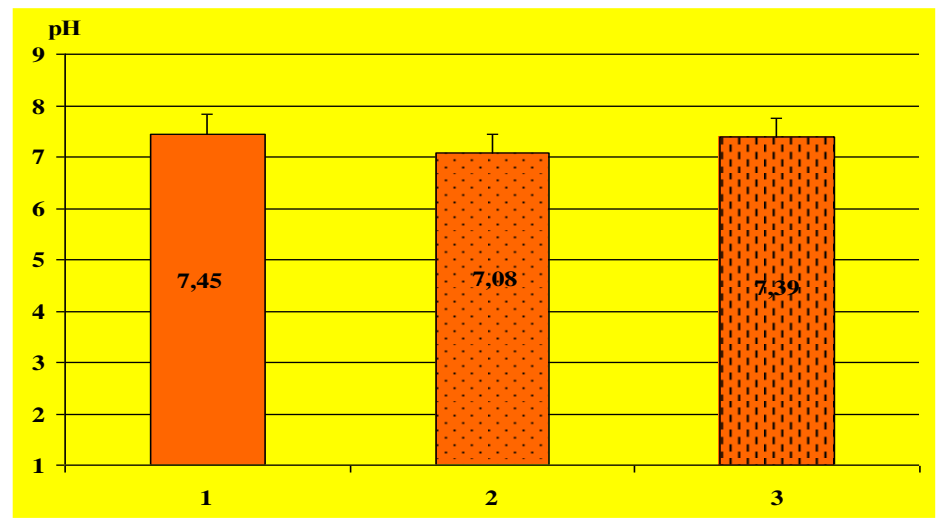

Note: on ordinate axis, blood $\mathrm{pH}$, on abscess axis: 1 - normal, 2 - hypoxia in 30 minutes, 2 - in hypoxia after 60 minutes.

Fig. 2. pH values in rat blood.

We see that the partial oxygen pressure (pO2) changed significantly compared with the control group (130 in normal; 114.25-75.8 $\mathrm{mmHg}$ after acute hypoxia). The increase of pOO2 in 2,2 and 3,5 times in comparison with control group, which indicates absence of compensatory processes of the acid-base state in blood of animals in respiratory units [19].

During various changes in the body, blood cells can function according to their properties. Initially, hypoxia showed a decrease in the number of erythrocytes by $8.9 \%$, and a recent increase of $17.14 \%$ after 60 minutes (in the control group $8.87 \pm 0.1 \mathrm{M} / \mathrm{mm} 3$ ). Normally, the number of leukocytes decreased to $6.79 \pm 0.2 \mathrm{~m} / \mathrm{mm} 3$, after acute hypoxia - to $2.62 \pm$ $0.1 \mathrm{~m} / \mathrm{mm} 3$, but after 60 minutes, an increase in leukocytes by $75.7 \%$ was observed in comparison with the control group (Fig. 3).

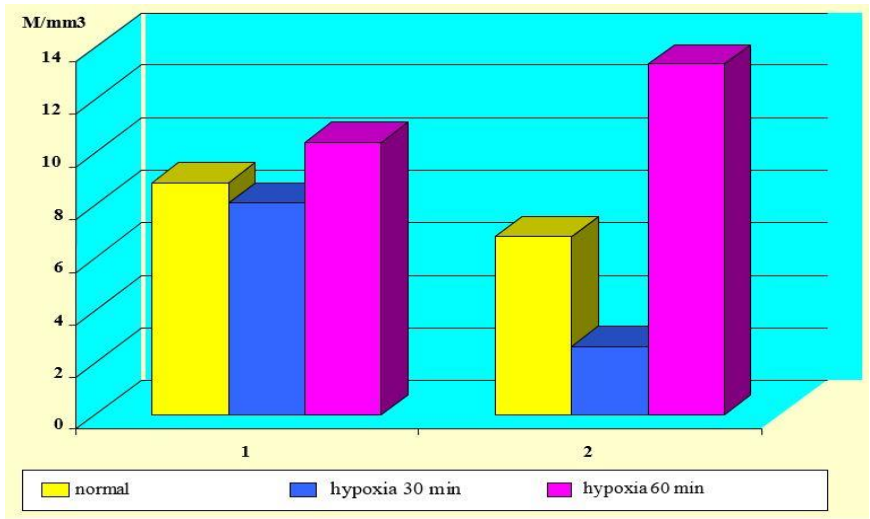

Note: on ascending ordinate: size of blood cells, M / mm3, on abscis: 1 - erythrocytes, 2 - leukocytes.

Fig. 3. Post-hypoxia of erythrocytes and leukocytes in rat blood. 
In the control group, lymphocyte rates increased by $59.08 \%$, and after single hypoxia increased 1.3 and 1.2 times ( $<<0.05$ ), respectively. Blood monocytes were $14.15 \%$ normal and 35.8 and $47.9 \%$ reduced after single hypoxia. After hypoxia, platelet count increased 2.3fold ( $\mathrm{p}<0.01$ ) compared with control group. Hemoglobin and hematocrit levels were elevated during single hypoxia (60 min.) Compared to baseline. The volume of hemoglobin in the blood of rats was 12.9 in the control group, and 16.7 and $15.8 \mathrm{~g} / \mathrm{dl}$ after ischemic reperfusion (Fig. 4).

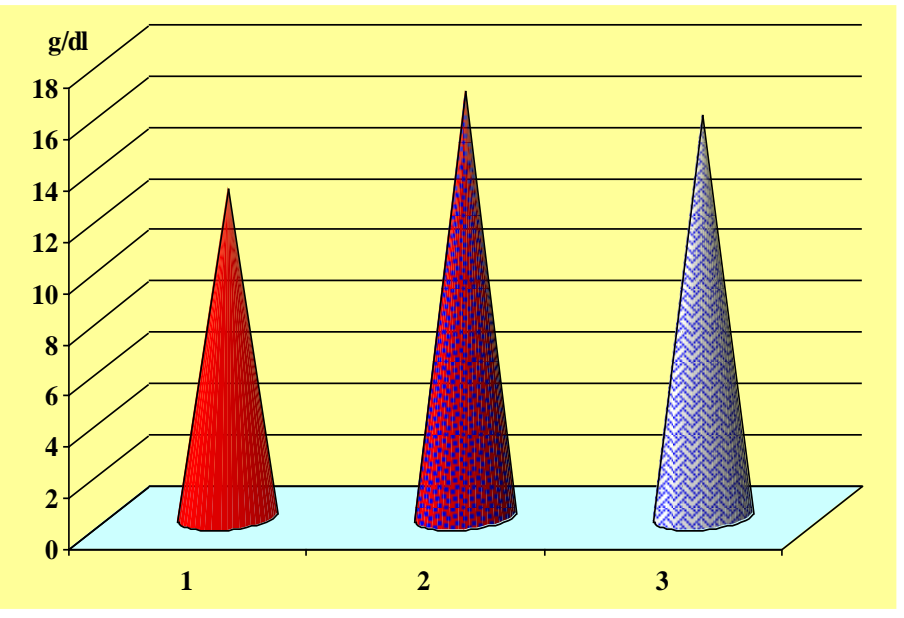

Note: on ordinate axis: hemoglobin content in blood, $\mathrm{g} / \mathrm{dl}$, on abscess growth: 1 - normal, 2 hypoxia in 30 minutes, 2 - in hypoxia after 60 minutes.

Fig. 4. Hemoglobin content in experimental hypoxia of rats.

According to the data obtained, rheological properties of blood, a decrease in $\mathrm{O} 2$ levels, which is a violation of the circulatory system in the hypoxia stage of the organs and circulatory disorders in the brain during oxygen deficiency, are disturbed in rats. As a result of changes in the amount of $\mathrm{O} 2$ in the blood, the blood supply to the tissues in the vessels decreases.

The data obtained show that the lack of oxygen in rats with a single hypoxia adversely affects the microcirculation of the blood system. At the same time during the hypoxia onetime increase in hematocrit and an increase in platelet count in the blood is observed, which leads to an increase in the blood-clotting properties of the blood as a result of hypoxia.

The physiological significance of proteins is very high. The cells occupy a leading position among the organic elements, accounting for $50 \%$ of the dry mass of cells. They have a very complex biological role. In practice, total protein levels were reduced after 60 minutes during acute hypoxia in the lymph and blood plasma: from $67.0 \pm 0.13$ to $62.11 \mathrm{~g} / 1$ in blood plasma, and $27.2 \pm 0.32$ to 22 in lymphoma, Reduced to $0 \pm 0.19 \mathrm{~g} / 1$. This, in turn, indicates a decrease in the transport function in the body (Fig. 5, Table 1). An increase in the level of urea and creatinine in the blood plasma of experimental animals was found, which indicates a violation of the activity of the animals during general experimental hypoxia.

In organisms, the proteins are renewed during the process of destruction and return. Belts do not collect like moths, but it is important that they are brought in with food. The decrease in total protein in the blood plasma of animals may be due to a decrease in protein synthesis in the nucleus accumbens, which has led to a decrease in the processes of filtration and 


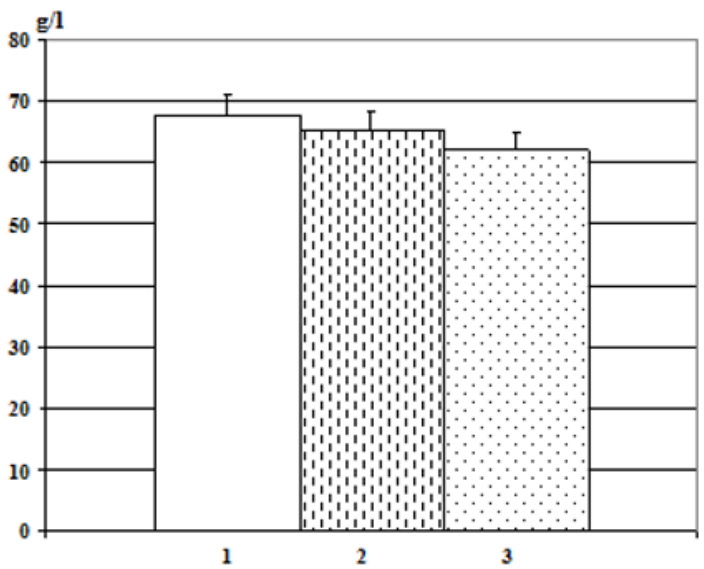

Note: on the ordinate axis: the total amount of protein in the blood plasma, $\mathrm{g} / \mathrm{l}$, on the abscess axis: 1 - normal condition, 2 - hypoxia in 30 minutes, 3 - in hypoxia after 60 minutes.

Fig. 5. Total protein levels in the blood plasma of rats with acute hypoxia.

absorption. The level of urea and creatinine in the blood plasma increased by 20 and $17 \%$, which, in turn, was associated with a decrease in protein formation and depletion (Table 1).

Table 1. Biochemical indicators of hypoxia activity in rat body.

\begin{tabular}{l|c|c|c}
\hline \multicolumn{1}{c|}{ Indicators } & Normal situation & $\begin{array}{c}\text { hypoxia for } 30 \\
\text { minutes }\end{array}$ & hypoxia 60 minutes \\
\hline $\mathrm{pO}_{2}$ & $70,00 \pm 3,17$ & $80,40 \pm 9,25^{*}$ & $91,50 \pm 3,37^{*}$ \\
\hline $\mathrm{pCO} 2$ & $50,00 \pm 2,12$ & $73,20 \pm 3,17^{*}$ & $52,50 \pm 5,32^{*}$ \\
\hline $\begin{array}{l}\text { Total protein in the blood } \\
\text { plasma, g / }\end{array}$ & $67,7 \pm 1,07$ & $65,2 \pm 1,32$ & $62,11 \pm 1,15^{*}$ \\
\hline $\begin{array}{l}\text { Total protein in the } \\
\text { lymph, g / }\end{array}$ & $42,5 \pm 1,18$ & $39,7 \pm 1,65^{*}$ & $36,1 \pm 1,89^{* *}$ \\
\hline $\begin{array}{l}\text { ALAT in blood plasma, } \\
\text { mkkat }\end{array}$ & $0,12 \pm 0,02$ & $0,48 \pm 0,08^{*}$ & $0,67 \pm 0,12^{* *}$ \\
\hline ALAT in lymph, mkkat & $0,13 \pm 0,09$ & $0,40 \pm 0,09^{*}$ & $0,60 \pm 0,07^{* *}$ \\
\hline $\begin{array}{l}\text { ASAT in the blood } \\
\text { plasma, mkkat }\end{array}$ & $0,13 \pm 0,02$ & $0,43 \pm 0,09^{*}$ & $0,58 \pm 0,07^{* *}$ \\
\hline $\begin{array}{l}\text { ASAT in the lymph, } \\
\text { mkkat }\end{array}$ & $0,14 \pm 0,02$ & $0,34 \pm 0,02^{*}$ & $0,51 \pm 0,02^{*}$ \\
\hline Plasma urea, mmol / & $5,44 \pm 0,12$ & $6,20 \pm 0,19^{*}$ & $6,56 \pm 0,11^{*}$ \\
\hline $\begin{array}{l}\text { Creatinine in the blood } \\
\text { plasma, mmol / }\end{array}$ & $59,44 \pm 3,35$ & $63,6 \pm 2,22^{*}$ & $69,96 \pm 3,54^{*}$ \\
\hline Note: in comparison with reliability group- $<<0,05^{*} ;-\mathrm{P}<0,01^{* *}$ & \\
\hline
\end{tabular}

Aminotransferases are found in all cells of the body, and transaminases have a predominantly clinical significance in the expression of the activity of alaninaminotransferases (ALAT) and aspartataminotransferases (ASAT). ALT is found in all parenchymal organs and ASAT in all tissues of the body. In our research, there was a decrease in plasma ALAT and AsAT levels by 3.5-4 times, respectively, with a decrease in the total protein in the blood plasma (ALAT -0.12 at baseline, AsAT - 0.13 mkkat). The increase in ALAT and ASAT in the blood indicates the strengthening of some destructive (destructive) processes in the liver, cardiovascular system (Fig. 6, Table 1). 
Therefore, in the case of short-term hypoxia, hemodynamic and lymphodynamic indicators will decrease and rheological parameters of lymph and blood will change. The blood and lymph systems play a key role in maintaining the equilibrium state of the internal environment with changes in the internal and external environment.

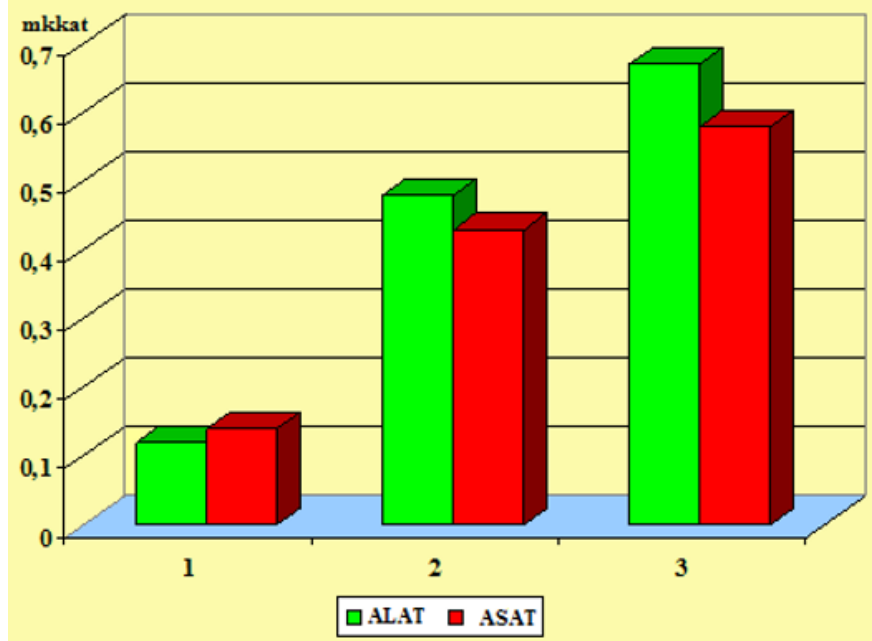

Note: on ordinate axis: level of aminotransferases in blood, mkkat, on abscis growth: 1 - normal state, 2 - hypoxia in 30 minutes, 3 - in hypoxia after 60 minutes.

Fig. 6. Amount of aminotransferases in experimental plasma hypoxia of rats.

The quantitative and qualitative composition of erythrocyte cells in humans and animals is characterized by a relatively high degree of stability under normal and pathological conditions, which is due to the constant action of the mechanisms of movement. In practice, after 30 minutes of experimental hypoxia, the time of blood and lymph clotting decreased by 33 and 30\%, respectively, and their viscosity increased by 15 and 10\% (Fig. 7).

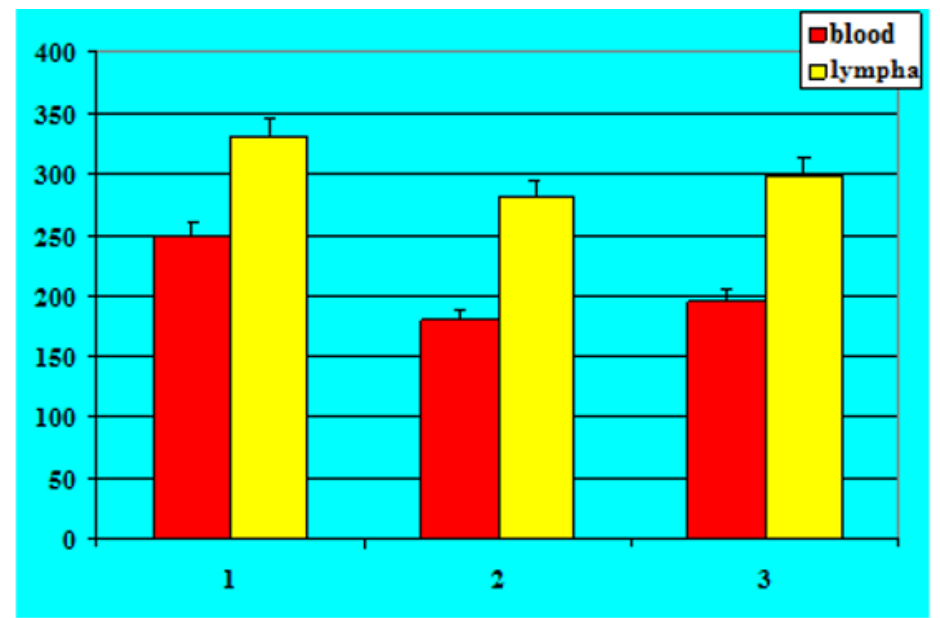

Note: on ordinate axis - time sec, on abscis axis - stages of practice: 1 - normal state, 2 - hypoxia in 30 minutes, 2 - hypoxia in 60 minutes.

Fig. 7. Stages of blood and lymph coagulation with short-term hypoxia of animals. 
In animals, after acute hypoxia, the coagulation of lymph and blood by the Sukharev method was 2.8 and 2.43 minutes, compared with the control group, which, in turn, increased the rate of clotting by $34-35 \%$, respectively. Indications of clotting velocity suggest that very severe changes in lymph and blood during ischemia and reperfusion increase the thrombogenic properties of blood (Fig. 8).

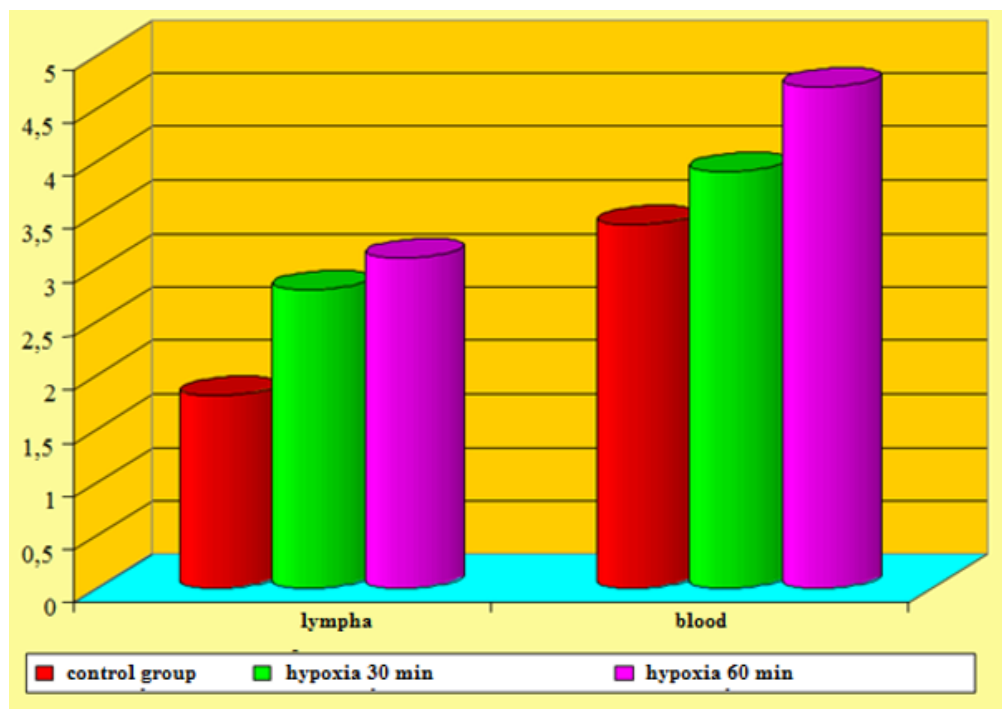

Note: expressed as a percentage of viscosity on ordinate axis, on abscissa axis: 1 - initial period, $100 \%$ taken, 2 - hypoxia after 30 minutes, 3 - hypoxia after 60 minutes.

Fig. 8. Blood and lymph viscosity during hypoxia in animals.

During hypoxia, lymph flow was reduced to $0.158 \pm 0.1 \mathrm{ml} / \mathrm{min}$. Animal blood coagulability was $183 \pm 3.6 \mathrm{~s}$, during the control periods, $248 \pm 4.4 \mathrm{~s}$. Lymph drainage was $288 \pm 7 \mathrm{sec}$, at initial stages $330 \pm 5.1 \mathrm{sec}$. During hypoxia, blood viscosity increases to 5.20 $\pm 0.20(\mathrm{P}<0.05 *)$, equal to $5.00 \pm 0.5$, in the lymph it is increased from $3.9 \pm 0.3$ to $5.35 \pm$ $0.5(\mathrm{P}<0.05 *)$ increased. In animals during the period of hypoxia there are deviations of rheological properties of lymph and blood. Compared to the control group, the viscosity of the lymph and blood increases, and its coagulation is accelerated. According to the data obtained, animals had an increased viscosity of the lymph and blood during hypoxia, which, in turn, indicates a thickening of the blood when regulating fluid in the body. Decreased lymph and blood clotting time, increased hematocrit, and an increase in the blood and lymph's thrombogenic properties as a result of oxygen deficiency in the body [15]. With hypoxia, the minute volume of blood and the frequency of contractions of the heart increase [16], and the blood pressure increases [17]. Increased blood pressure during hypoxia is considered as a result of the reflex effect of vasoconstriction [18]. In hypoxia, people may have brain hemorrhage, impaired heart or kidney function (anuria), as well as other conditions [20].

In recent years the physiological, structural, functional and biochemical features of the organism to some degree and specific to various diseases are being actively studied. In accordance with the goals and in our research, the functional status of the lymph and blood system was examined: in particular, the lymphatic drainage and lymphatic structure, biochemical and rheological properties. Scientific literature has shown that hematocrit depletion with oxygen deficiency in the body can be caused by a violation of plasma volume and that there are changes in blood cell elements. At the same time, with hypoxia, simultaneous violation of carbohydrate intake, there is a change in proteins. 
In our opinion, we noticed that during experimental hypoxia, there were minor changes in the body, in turn, the blood and lymphatic system functioned to have a compensatory and compensatory role in the case of oxygen deficiency in the body, and an increase in the activity of leukocytes in the blood was observed. The obtained results are evidence of the involvement of the lymphatic system in various changes in the body of animals and in the regulation of the balance of the organism.

\section{Conclusions}

It has been shown that during acute hypoxia of animals, in particular, compression of the carotid artery has a negative effect on biochemical blood parameters, which in turn led to a violation of metabolism due to a lack of oxygen in the blood vessels. As a result of a decrease in the total protein content in the blood supply by $11 \%$, when compared with the batch, the excretion of the final product of aqueous alcohol, the bladder of the bladder, was broken down and destroyed. The level of ALAT and ASAT in the blood plasma is increased by 3.54 times, respectively, which indicates an increase in cytological processes in the cardiovascular system and organs. In experimental hypoxia in animals, the limb is accompanied by a violation of the rheological properties of lymph and blood, an increase in the lymph and blood clotting velocity and an increase in their viscosity. Indicates that the $\mathrm{pH}$ of the blood changes in the direction of acidosis during acute hypoxia in animals. Blood $\mathrm{pH}$ decreased by $4.9 \%$ during the first 30 minutes and $0.8 \%(\mathrm{p}<0.05)$ during the first 30 minutes ( $\mathrm{pH} 7.45$ in the control group). Due to the increase in the thrombogenic properties of blood and lymph, the phenomenon of acidosis is observed, and the biochemical parameters change.

In acute hypoxia there is a violation of the rheological properties of lymph and blood, as well as red blood cells, erythrocytes, leukocytes and platelets - platelets. Due to the increased thrombogenic properties of blood and lymph, the phenomenon of acidosis and the variability of biochemical parameters are observed. Following the hypoxia model,it has been shown that animals have a negative effect on the internal environment, lymphodynamics, and the biochemical composition of blood and lymph. By the method of rheography studies of blood supply in the trunk during acute experimental hypoxia, it was found that the change in the linear frequency of the blood flow in the main vein, the decrease in the activity of the lymphatic flow and lymph nodes are slowed down. This is explained by a violation of the peceptoplasm in its lymph nodes and vessels.

\section{References}

1. S.N. Abdrechov, L.E. Bulekbaeva, G.A. Demchenko, Russian Journal of Physiology named after I. M. Sechenov of the Russian Academy of Sciences, 97(5), 509-514 (2011)

2. U.I. Borodin, B.N. Grigorev, Lymph node with circulatory disorders, 268 (Novosibirsk, 2000)

3. T.A. Astashova, E.S. Kazakova, Problems of lymphology and interstitial mass transfer: Mater. Int. conf., 230-233 (2014)

4. S.N. Abdreshov, L.E. Bulekbaeva, G.A. Demchenko, Bulletin of Experimental Biology and Medicine, 159(1), 32-34 (2015)

5. F.Z. Meerson, 272 (Medicine, Moscow, 2001)

6. M.K. Murzahmetova, 232 (Almaty, 2001)

7. S.N. Abdreshow, G.A. Demshenko, Bulletin of Experimental Biology and Medicine, 148(3), 403-405 (2009)

8. J. Kirheby Ole, S. Kutszsche, R. RisoeCulie R, J. ClinNeuroschi, 7(6), 531-538 (2000)

9. A.A. Lev, 323 (Nauka, SPb., 2005) 
10. I.E. Bruce Jason, C. Austin, J. Physiol. Proc., 523, 118-119 (2000)

11. D.Y. Saenko, I.V. Saenko, M.P. Shestakov, A.M. Ivanov, I.B. Kozlovskaia, Aviakosm. Ekolog. Med., 34(5), 6-10 (2000)

12. L. Packer, Am. J. Clin. Nutr., 53(4), 1050-1055 (2000)

13. H. Miranda, E. Viikari-Juntura, R. Martikainen et al., Spine, 27(10), 1102-1109 (2002)

14. G.M. Russell, S.M. Roach, Med J Aust, 176(8), 367-370 (2002)

15. R.J. Reiter, News in physiological sciences, 15(10), 246-250 (2000)

16. T. Oxman, M. Arad, R. Klein, N. Avazov, Amer. Y. Physiol, 273(4), 1707-1712 (2001)

17. S.N. Abdreshov, N.A. Akhmetbaeva, G.K. Atanbaeva, A.T. Mamataeva, U.B. Nauryzbai, Bulletin of Experimental Biology and Medicine, 168(2), 295-299 (2019)

18. K.A. Saparov, Z.B. Yessimsiitova, B.A. Abdullayeva, (...), G.K. Atanbayeva, S.N. Abdreshov, Journal of Pharmacy and Nutrition Sciences, 9(2), 101-103 (2019)

19. A.A. Mautenbaev, G.K. Atanbaeva, E.A. Kyrbassova, (...), G.I. Issayev, Z.T. Abdrassulova, Journal of Pharmaceutical Sciences and Research, 10(12), 3214-3215 (2018)

20. D. Johnson Christopher, M. Areher Joanne, Abstr Jt. SciMut. Physiol. Soc. With Brit. Pharmacology Soc. Southampton, 8-11 (2008) 